\title{
Exposure of $\mathrm{Gd}_{2} \mathrm{O}_{3}$-alanine and $\mathrm{Gd}_{2} \mathrm{O}_{3}$-ammonium tartrate ESR dosimeters to thermal neutrons: Experiments and Monte Carlo simulations
}

\author{
M. Marrale ${ }^{\mathrm{a}, \mathrm{c}, *}$, G. Gennaro ${ }^{\mathrm{a}}$, M. Brai ${ }^{\mathrm{a}, \mathrm{c}}$, S. Basile ${ }^{\mathrm{a}, \mathrm{c}}$, A. Bartolotta ${ }^{\mathrm{b}, \mathrm{c}}$, M.C. D’Oca ${ }^{\mathrm{b}}$ \\ ${ }^{a}$ Dipartimento di Fisica e Tecnologie Relative, Università di Palermo, Viale delle Scienze, Edificio 18, 90128 Palermo, Italy \\ ${ }^{\mathrm{b}}$ Dipartimento Farmacochimico Tossicologico e Biologico, Università di Palermo, Via Archirafi 32, 90123 Palermo, Italy \\ ${ }^{\mathrm{c}}$ Unità CNISM, Palermo, Italy
}

\begin{abstract}
Electron spin resonance solid state dosimetry with alanine and ammonium tartrate pellets is a valuable tool in both medical and industrial applications when dealing with photon and charged particle beams. Its use in neutron beams is limited by the low nuclear cross section values of atoms in the pellets. Addition of boron $\left({ }^{10} \mathrm{~B}\right)$ or gadolinium $\left(\mathrm{Gd}_{2} \mathrm{O}_{3}\right)$, known to have high neutron capture cross sections, has been proposed to improve neutron sensitivity.

In this paper we present the results of an experimental study concerning neutron sensitivity vs. gadolinium concentration in dosimeter mixtures, with the aim of optimizing mixture composition and maximizing the electron spin resolution (ESR) signal. For gadolinium concentrations up to about $25 \%$, the dosimeter sensitivity increases with concentration. A saturation value is reached afterwards because of the smaller presence of the main ESR sensible compounds (i.e. alanine or ammonium tartrate).

Monte Carlo techniques have been applied and comparisons between computational and experimental results have been carried out. (C) 2007 Elsevier Ltd. All rights reserved.
\end{abstract}

Keywords: ESR dosimetry; Alanine; Ammonium tartrate; Monte Carlo simulation

\section{Introduction}

Various progresses have been made in electron spin resonance (ESR) dosimetry for applications ranging from radiation sterilization to radiation therapy (Ikeya, 1993; Regulla, 2000). This technique allows to determine the radiation dose by measuring the total concentration of free radicals produced by ionizing radiation. Developing new materials for better radiation response is one of the most interesting target of the ESR dosimetry research (Ikeya et al., 2000; Lund et al., 2002). Various materials have been tested indeed in ESR dosimetry; among them, alanine (Regulla and Deffner, 1982), ammonium tartrate (AT) (Olsson et al., 1999; Olsson, 2001; Bartolotta et al., 2001; Marrale et al., 2006), lithium lactate (Hassan et al.,

\footnotetext{
* Corresponding author at: Dipartimento di Fisica e Tecnologie Relative, Università di Palermo, Viale delle Scienze, Edificio 18, 90128 Palermo, Italy. Fax: +390916615063 .

E-mail address: marrale@difter.unipa.it (M. Marrale).
}

1998), blends of amino acids (Bartolotta et al., 1999), formates and dithionates (Lund et al., 2005) are widely used.

Often these materials do not have high sensitivity to thermal neutrons and, therefore, they are unable to accurately measure neutron fluence because of the low neutron capture cross section of their nuclei. This leads to weak ESR signal for samples exposed to thermal neutrons and it is very difficult to measure the neutron beam properties. The addition of nuclei with high neutron capture cross section is a method for improving the interactions with thermal neutrons. Some nuclei recently added for enhancing sensitivity of ESR dosimeters to thermal neutrons are: ${ }^{6} \mathrm{Li}$ (Lund et al., 2004; Herrera et al., 2005), ${ }^{10} \mathrm{~B}$ (Galindo and Ureña-Nuñez, 1993; Ureña-Nuñez et al., 1998, 1999; Bartolotta et al., 2004) and gadolinium (Marrale et al., 2007; Brai et al., 2007a, b)

Monte Carlo simulations can be a very useful tool for testing new materials. Nowadays, various applications of Monte Carlo simulation are carried out to obtain information about the radiation transport and the energy deposition for various radiation 
beams and for various irradiation set-ups. Usually, the simulation is exploited since it allows to predict some results without performing any experiment which could be very expensive, time consuming or, as in case of radiotherapy treatment, dangerous to health. For radiotherapy, the simulations are widely used to calculate dose distributions for treatment planning of photon, electron and proton irradiation (Ma and Jiang, 1999; Mobit et al., 1996; Paganetti, 2006). The simulations are also very widespread for calculation of various detection techniques with various sensible materials (Ma and Nahum, 1993). In the last years, along with the progresses of the ESR dosimetry, the response simulation of various materials has aroused great interest. Usually, the comparison of experimental data with Monte Carlo computations has been performed in order to better understand the response mechanisms and to get information in the dosimeter quality (Zeng et al., 2004, 2005; Zeng and McCaffrey, 2005; Fattibene et al., 2003).

In this paper we analyze the response to thermal neutrons of ESR pellets of alanine and AT added with gadolinium. In particular, we study the sensitivity improvement vs. gadolinium content inside the dosimeter. The experimental results are compared to Monte Carlo simulations in order to achieve information about the energy deposition inside the volume of these ESR detectors. This computational analysis is aimed at knowing the ability of the Monte Carlo technique to accurately describe the ESR response of these dosimeters with and without additive after the exposure to neutrons.

\section{Materials and methods}

\subsection{Solid state ESR pellets preparation procedure}

Solid state pellets for ESR dosimetry prepared using a blend of L- $\alpha$-alanine (Fluka, Buchs, Switzerland) or AT (Carlo Erba, Italy) and gadolinium-oxide (Aldrich Chem. Co) in known weight proportions.

The pellets were realized following a procedure previously optimized (Bartolotta et al., 1999) by pressing a blend of $94 \%$ of the appropriate active material (alanine with $\mathrm{Gd}_{2} \mathrm{O}_{3}$ or AT with $\mathrm{Gd}_{2} \mathrm{O}_{3}$ ), $5 \%$ of polyethylene (Polysciences, $\mathrm{MW}=700$ ) as binder, and $1 \%$ of magnesium stearate (Carlo Erba, Milano, Italy) as lubricant. Up to $47 \%$ of gadolinium was present in the doped pellets.

The pellets had total mass of $28 \mathrm{mg}$, diameter of $4.0 \mathrm{~mm}$ and thickness linearly decreasing with $\mathrm{Gd}$ concentration from 2.5 to $1.9 \mathrm{~mm}$ for alanine pellets and from 2.2 to $1.7 \mathrm{~mm}$ for AT pellets. This is because the high atomic number of gadolinium $(Z=64)$ increases the density of the pellets and the thickness is reduced as a consequence of the gadolinium addition.

\subsection{Irradiation}

Thermal neutron irradiations were carried out at the TAPIRO reactor, ENEA Casaccia (Rome). Since TAPIRO is a source of fast neutrons, the facility HYTHOR (HYbrid Thermal spectrum sHifter TapirO Reactor) was used to obtain a beam of thermal neutrons (Esposito et al., 2007). Teflon cylindrical dosimeter holders were used for irradiations. Four dosimeters were placed in suitable small cavities inside holders, and irradiated inside thermal column of reactor at neutron fluence of $(18.8 \pm 0.2) \times$ $10^{12} \mathrm{~cm}^{-2}$.

\subsection{ESR measurements}

The ESR measurements were recorded at room temperature with a Bruker ECS 106 spectrometer equipped with a $\mathrm{TE}_{102}$ rectangular cavity and operating in the $\mathrm{X}$ band at approximately 9.75 GHz. The first derivative of the ESR alanine (AT) absorption spectrum was recorded with the following parameters: field sweep of 20 (10) mT; modulation amplitude of $0.25(0.28) \mathrm{mT}$; microwave power of $6.32(1.59) \mathrm{mW}$.

The peak-to-peak signal amplitude $h_{\mathrm{pp}}$ of the central line was measured and used as dosimetric parameter (Regulla and Deffner, 1982). Since we observed a dependence of the ESR signal on the orientation of each pellet inside the resonant cavity as reported in literature (Kojima et al., 1995), we read out all samples at four orientations with respect the cylindrical axis of the tube and we corrected the $h_{\mathrm{pp}}$ by the fractional weight content of sensitive material in the sample.

The dosimeters containing gadolinium oxide show the ESR signal of alanine or AT superimposed to that of $\mathrm{Gd}_{2} \mathrm{O}_{3}$, which is very wide $(\sim 270 \mathrm{mT})$ and centered in the $g \sim 2$ region field (Brai et al., 2007a, b). The contribution of $\mathrm{Gd}_{2} \mathrm{O}_{3}$ signal in the magnetic field range swept is linear and it was subtracted from each spectrum. The $h_{\mathrm{pp}}$ for these dosimeters was measured after this baseline elimination. For each gadolinium concentration three dosimeters were measured at four different orientations to obtain an average value.

\subsection{Monte Carlo simulation}

The Monte Carlo N-particle-MCNP5 (Briesmeister, 2000) radiation transport code was used to perform the calculations in this work. Fig. 1 shows the geometrical configuration of neutron source and target as set in the simulations. Neutron beam features, such as the energy spectrum and the geometry of the source, were taken into account. The neutron source is a disk source in $x-y$ plane, $1 \mathrm{~cm}$ diameter, coaxial with $z$ axis and at a distance of $5 \mathrm{~mm}$ from dosimeter holder. The beam is unidirectional with $90 \%$ thermal and 10\% from epithermal and fast neutron contamination. To simulate the various materials the neutrons passes through, we chose to consider them as parallel layers. Since the pellet holder has axial symmetry, all layers are uniaxial cylinders and the source is a disk with the center on these symmetry axes. The presence of the gadolinium oxide inside the pellet was taken into account by locating thin $100 \mu \mathrm{m}$ layers (the average size of the gadolinium oxide microcrystals in the mixture) between two layers of sensitive material (alanine or AT). The pellets with higher gadolinium concentrations were composed of a larger number of gadolinium oxide layers. Water was chosen to simulate plastic dosimeter holder. Neutrons undergo interactions with air, water and the nuclei of various alternating layers of sensitive materials and gadolinium 


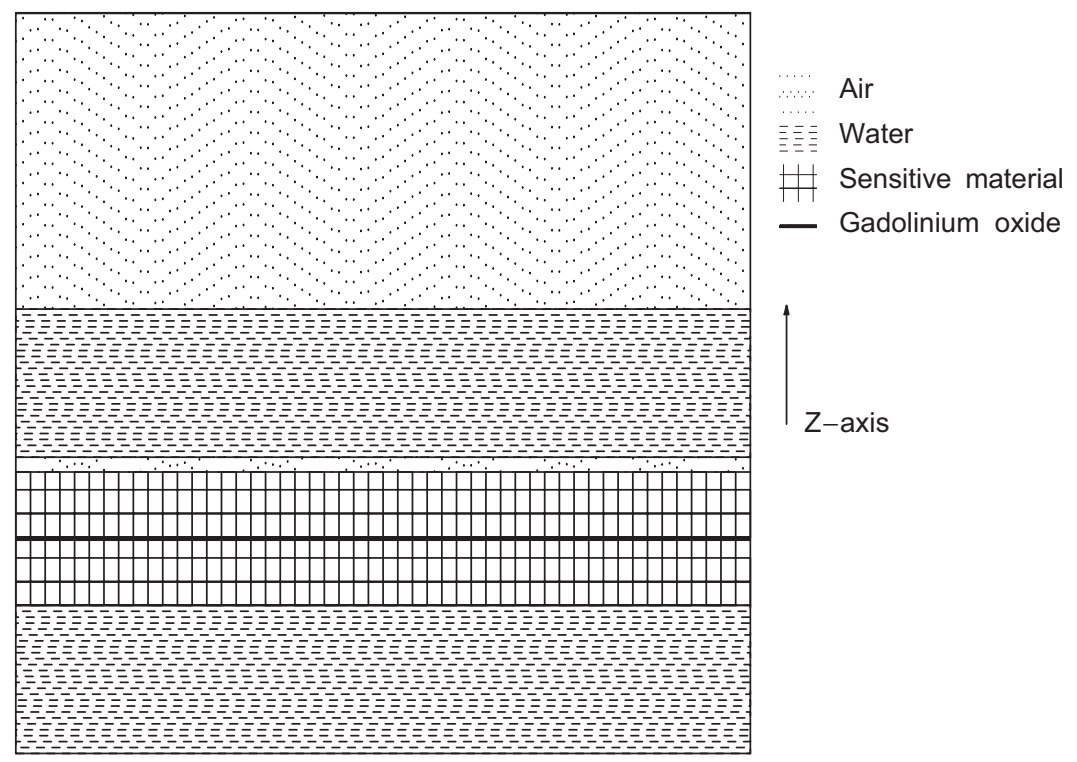

Fig. 1. Example of the irradiation and target geometry used for Monte Carlo simulation used in the case of single gadolinium layer. Multiple gadolinium layers were considered for high concentrations. The beam direction is from top to bottom.

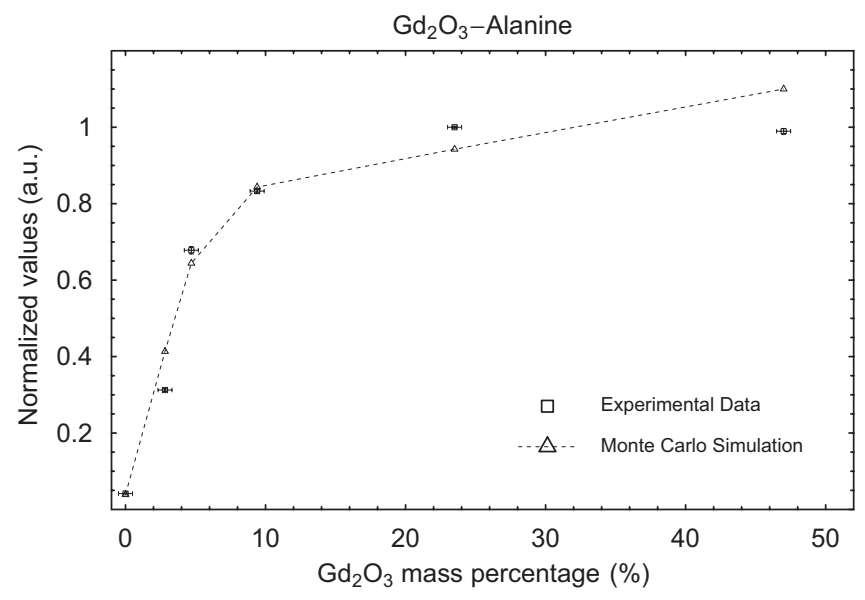

Fig. 2. Experimental data (normalized to their maximum value) and Monte Carlo simulation for alanine exposed to neutrons.

oxide. The quantity we are interested in is the average amount of energy deposition per unit mass of the sensitive material. We compared the values of this quantity with the experimental ESR signal data of both organic compounds analyzed.

\section{Results and discussion}

Figs. 2 and 3 show the ESR signal amplitude due to neutrons per unit mass vs. the gadolinium oxide percentage content for alanine and AT dosimeters, respectively. Data are normalized to their maximum value. Examples of ESR spectra, from which the peak-to-peak amplitudes have been extracted, can be found in Brai et al. (2007a, b).

For low $\mathrm{Gd}_{2} \mathrm{O}_{3}$ content the ESR signal peak-to-peak amplitude is proportional to the amount of gadolinium oxide inside

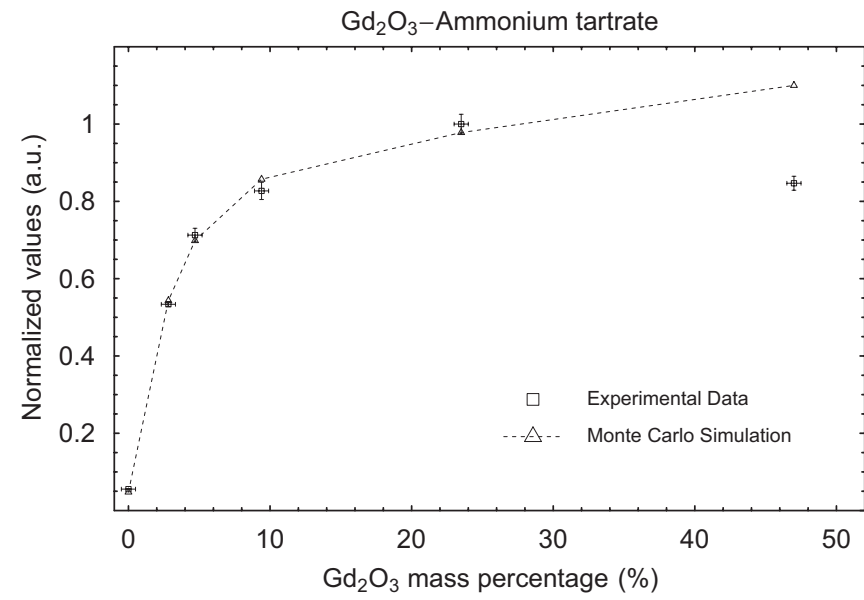

Fig. 3. Experimental data (normalized to their maximum value) and Monte Carlo simulation for ammonium tartrate exposed to neutrons.

the dosimeter. The ESR signal increases with the content of additive in blend and reaches its maximum value and then decreases. The signal amplitude is maximized for both organic compounds when the mass percentage of gadolinium oxide is about $25 \%$ of the total mass of the dosimeter. The signal enhancement is of about 30 times for alanine and of about 20 times for AT.

This behavior is due to two competing factors which contribute to the ESR signal. As the gadolinium content is increased, the number of targets per unit volume becomes larger and the macroscopic neutron cross section increases. Therefore, the probability of interaction between thermal neutrons and nuclei becomes larger so raising the number of free radicals. On the other hand, since all dosimeters have the same mass, an increment of the mass percentage of gadolinium oxide brings 
about the reduction of the sensitive compound (either alanine or AT) content inside the pellet. Therefore, even though the number of targets is raised with high gadolinium presence, the pellets have a lower sensitivity because the total number of molecules able to produce the free radicals is reduced.

We performed the Monte Carlo simulations with the accurate definitions of the irradiation set-up and dosimeter materials and geometry. Figs. 2 and 3 show a good agreement between experiments and Monte Carlo computations for low $\mathrm{Gd}_{2} \mathrm{O}_{3}$ concentrations. This result highlights that the parameters and inputs provided for the simulations allow a good description of the system. In particular, this proves that the ESR signal is proportional to the amount of energy released inside the dosimeters.

At high gadolinium concentrations the simulated values differs from the experimental values. This effect is very evident for the highest $\mathrm{Gd}_{2} \mathrm{O}_{3}$ concentration studied (47\%). A possible explanation of this difference is a saturation effect in free radicals of the sensitive materials. The energy released inside alanine or AT is very high but not a so high number of molecules from which free radicals can be produced is present. Furthermore, high spatial concentrations of free radicals could take place and this situation could lead to recombination phenomena. Therefore, the ESR signal per unit mass decreases with increasing gadolinium content inside the pellet. However, this saturation effect is not taken into account in the Monte Carlo simulation for which the released energy keeps increasing with gadolinium concentration.

\section{Conclusions}

In the present work, we investigated how the ESR response of pellets of alanine and AT exposed to thermal neutrons varies with gadolinium oxide concentration. We found that the addition of the gadolinium increases the sensitivities to thermal neutrons for both organic compounds. We carried out Monte Carlo simulations for obtaining information about the reliability of this powerful tool in predicting the response enhancement achievable with use of suitable additive. We found that the Monte Carlo computed values well follow the experimental data for low gadolinium level inside the dosimeter; whereas at high gadolinium concentration the difference between simulated and experimental data is larger because of the saturation effect in free radicals of sensitive materials. In conclusion, the Monte Carlo simulation can be chosen as a useful tool to predict the ESR response of organic compounds after the addition of suitable nuclei for enhancing the sensitivity to various radiation beams.

\section{Acknowledgments}

The research described in this paper was partially supported by the Università di Palermo and "Dottorato in Fisica Applicata" of the Università di Palermo. The authors are grateful to Giancarlo Rosi of the ENEA Casaccia, Rome, for the neutron irradiations and to Marcello Mirabello for technical support.

\section{References}

Bartolotta, A., Brai, M., Caputo, V., De Caro, V., Giannola, L.I., Rap, R., Teri, G., 1999. ESR solid state dosimetry: behaviour of various amino-acids and blend preparation procedures. Radiat. Prot. Dosim. 84, 293-296.

Bartolotta, A., D’Oca, M.C., Brai, M., Caputo, V., De Caro, V., Giannola, L.I., 2001. Response characterization of ammonium tartrate solid state pellets for ESR dosimetry with radiotherapeutic photon and electron beams. Phys. Med. Biol. 46 (2), 461-471.

Bartolotta, A., D’Oca, M.C., Lo Giudice, B., Brai, M., Borio, R., Forini, N., Salvatori, P., Manera, S., 2004. Combined TL and ${ }^{10} \mathrm{~B}$-alanine ESR dosimetry for BNCT. Radiat. Prot. Dosim. 110, 627-630.

Brai, M., Gennaro, G., Marrale, M., Bartolotta, A., D’Oca, M.C., 2007a. ESR response to $\gamma$-rays of alanine pellets using $\mathrm{B}(\mathrm{OH})_{3}$ or $\mathrm{Gd}_{2} \mathrm{O}_{3}$. Appl. Radiat. Isot. 65, 435-439.

Brai, M., Gennaro, G., Marrale, M., Tranchina, L., Bartolotta, A., D’Oca, M.C., 2007b. ESR response to ${ }^{60}$ Co $\gamma$-rays of ammonium tartrate pellets using $\mathrm{Gd}_{2} \mathrm{O}_{3}$ as additive. Radiat. Meas. 42, 225-231.

Briesmeister, J.F., (Ed.), 2000. Los Alamos National Laboratory report LA13709-M.

Esposito, J., Rosi, G., Agosteo, S., 2007. The new hybrid thermal neutron facility at TAPIRO reactor for BNCT radiobiological experiments. Radiat. Prot. Dosimetry, in press, doi:10.1093/rpd/ncm015.

Fattibene, P., Angelone, M., Pillon, M., De Coste, V., 2003. Tooth enamel dosimetric response to $2.8 \mathrm{MeV}$ neutrons. Nucl. Instrum. Meth. B 201, 480-490.

Galindo, S., Ureña-Nuñez, F., 1993. EPR signal enhancement of alanine irradiated with thermal neutrons. Radiat. Res. 133, 387-389.

Hassan, G.M., Ikeya, M., Toyoda, S., 1998. Lithium lactate as an ESR dosimeter. Appl. Radiat. Isot. 49, 823-828.

Herrera, E., Ureña-Nuñez, F., Delfín Loya, A., 2005. Lithium carbonate $\left(\mathrm{Li}_{2} \mathrm{CO}_{3}\right)$ as a material for thermal neutron fluence measurements. Appl. Radiat. Isot. 63, 241-246.

Ikeya, M., 1993. New Applications of Electron Spin Resonance. World Scientific Publishing, Singapore.

Ikeya, M., Hassan, G.M., Sasaoka, H., Kinoshita, Y., Takaki, S., Yamanaka, C., 2000. Strategy for finding new materials for ESR dosimeters. Appl. Radiat. Isot. 52, 1209-1215.

Kojima, T., Kashiwazaki, S., Tachibana, H., Tanaka, R., Desrosiers, M.F., McLaughlin, W.L., 1995. Orientation effects on ESR analysis of alaninepolymer dosimeters. Appl. Radiat. Isot. 46, 1407-1411.

Lund, A., Olsson, S., Bonora, M., Lund, E., Gustafsson, H., 2002. New materials for ESR dosimetry. Spectrochim. Acta Part A 58, 1301-1311.

Lund, E., Gustafsson, H., Danilczuk, M., Sastry, M.D., Lund, A., 2004. Compounds of ${ }^{6} \mathrm{Li}$ and natural $\mathrm{Li}$ for EPR dosimetry in photon/neutron mixed radiation fields. Spectrochim. Acta Part A 60, 1319-1326.

Lund, E., Gustafsson, H., Danilczuk, M., Sastry, M.D., Lund, A., Vestad, T.A., Malinen, E., Hole, E.O., Sagstuen, E., 2005. Formates and dithionates: sensitive EPR-dosimeter materials for radiation therapy. Appl. Radiat. Isot. 62, 317-324.

Ma, C.M., Jiang, S.B., 1999. Monte Carlo modelling of electron beams from medical accelerators. Phys. Med. Biol. 44, R157-R189.

Ma, C.M., Nahum, A.E., 1993. Dose conversion and wall correction factors for Fricke dosimetry in high-energy photon beams: analytical model and Monte Carlo calculations. Phys. Med. Biol. 38, 93-114.

Marrale, M., Brai, M., Triolo, A., Bartolotta, A., D’Oca, M.C., 2006. Power saturation of ESR signal in ammonium tartrate exposed to ${ }^{60} \mathrm{Co} \gamma$-ray photons. Electrons and Protons. Radiat. Res. 166, 802-809.

Marrale, M., Brai, M., Gennaro, G., Triolo, A., Bartolotta, A., D’Oca, M.C., Rosi, G., 2007. Alanine blends for ESR measurements of thermal neutron fluence in a mixed radiation field. Radiat. Prot. Dosim., in press, doi:10.1093/rpd/ncm 128 .

Mobit, P., Mayles, P., Nahum, A.E., 1996. The quality dependence of LiF TLD in megavoltage photon beams: Monte Carlo simulation and experiments. Phys. Med. Biol. 41, 387-398.

Olsson, S., 2001. ESR Dosimetry in the radiation therapy dose range. Linköping Medical Dissertations No. 701, Linköping, Sweden. 
Olsson, S.K., Bagherian, S., Lund, E., Carlsson, G.A., Lund, A., 1999. Ammonium tartrate as an ESR dosimeter material. Appl. Radiat. Isot. 50, 955-965.

Paganetti, H., 2006. Monte Carlo calculations for absolute dosimetry to determine machine outputs for proton therapy fields. Phys. Med. Biol. 51, 2801-2812.

Regulla, D., 2000. From dating to biophysics - 20 years of progress in applied spectroscopy. Appl. Radiat. Isot. 52, 1023-1030.

Regulla, D.F., Deffner, U., 1982. Dosimetry by ESR spectroscopy of alanine. Int. J. Appl. Radiat. Isot. 33, 1101-1114.

Ureña-Nuñez, F., Galindo, S., Azorin, J., 1998. An alanine-boron compound for thermal neutron fluence measurements. Part 1: synthesis and development. Appl. Radiat. Isot. 49, 1657-1664.
Ureña-Nuñez, F., Galindo, S., Azorin, J., 1999. An alanine-boron compound for thermal neutron fluence measurements. Part 2: EPR response. Appl. Radiat. Isot. 50, 763-767.

Zeng, G.G., McCaffrey, J.P., 2005. The response of alanine to a $150 \mathrm{keV}$ X-ray beam. Radiat. Phys. Chem. 72, 537-540.

Zeng, G.G., McEwen, M.R., Rogers, D.W.O., Klassen, N.V., 2004. An experimental and Monte Carlo investigation of the energy dependence of alanine/EPR dosimetry: I. Clinical X-rays beams. Phys. Med. Biol. 49, 257-270.

Zeng, G.G., McEwen, M.R., Rogers, D.W.O., Klassen, N.V., 2005. An experimental and Monte Carlo investigation of the energy dependence of alanine/EPR dosimetry: II. Clinical electron beams. Phys. Med. Biol. 50, 1119-1129. 